\title{
Why understanding-why is contrastive
}

\author{
Miguel Egler ${ }^{1}$
}

Received: 3 June 2020 / Accepted: 22 January 2021 / Published online: 9 February 2021

(c) The Author(s) 2021

\begin{abstract}
Contrastivism about interrogative understanding is the view that ' $\mathrm{S}$ understands why $p$ ' posits a three-place epistemic relation between a subject $\mathrm{S}$, a fact $p$, and an alternative to $p, q$. This thesis stands in stark opposition to the natural idea that a subject $\mathrm{S}$ can be said to understand why $p$ simpliciter. I argue that contrastivism offers the best explanation for the fact that evaluations of the form ' $\mathrm{S}$ understands why $p$ ' vary depending on the alternatives to $p$ under consideration. I also show that contrastivism offers valuable resources with which to explain the gradability of interrogative understanding attributions, as well as the sensitivity of these attributions to the perceived degree of epistemic demandingness of different contexts.
\end{abstract}

Keywords Understanding · Understanding-why · Contrastivism · Gradability · Context-sensitivity

\section{Introduction}

Interrogative understanding is the epistemic state ascribed by claims of the form ' $\mathrm{S}$ understands why p'-e.g., 'Mary understands why the Earth revolves around the Sun' or 'Quentin understands why Venezuela is in crisis'. In this paper, I argue for contrastivism about interrogative understanding. That is, I defend the following thesis:

Ternary 'Understands why' posits a three-place epistemic relation of understanding-why between a subject $\mathrm{S}$, a fact $p$, and an alternative to $p, q-\mathrm{i}$.e., U$\operatorname{why}_{(S, p, q)}$.

Ternary stands in stark opposition to the natural idea that we can analyse claims of the form ' $\mathrm{S}$ understands why $p$ ' in terms of a two-place epistemic relation holding between a subject $\mathrm{S}$ and a fact $p$. That is:

Binary 'Understands why' posits a two-place epistemic relation of understandingwhy between a subject $\mathrm{S}$ and a fact $p$-i.e., $\mathrm{U}$-why $(S, p)$.

\footnotetext{
$凶 \quad$ Miguel Egler

m.egler@tilburguniversity.edu

1 Department of Philosophy, Tilburg University, Tilburg, The Netherlands
} 
My main contention is that Ternary offers the best account of a notable pattern in our attributions of interrogative understanding: namely, their contrast-sensitivity. This refers to a natural shift in our evaluation of claims of the form ' $\mathrm{S}$ understands why $p$ ' depending on which alternatives to $p$ are under consideration. In addition, I demonstrate that Ternary allows us to account for the gradability of interrogative understanding attributions, as well as the sensitivity of these attributions to the perceived degree of epistemic demandingness of different contexts. In light of these considerations, I argue that there are good reasons to endorse Ternary.

Here is a detailed plan of the paper. I first give a brief overview of recent inquiry into the nature of interrogative understanding (Sect. 2). I then show that at least some interrogative understanding ascriptions are contrast-sensitive and propose that such attributions effectively express claims of the form 'S understands why $p$ rather than $q$ ' (Sect. 3). In the subsequent section (Sect. 4), I explain that Ternary naturally accommodates for such interrogative understanding ascriptions. I then argue that the most plausible readings of Binary cannot account for the contrast-sensitivity of interrogative understanding ascriptions (Sect. 5). Finally, I consider and reject three objections to Ternary (Sect. 6).

\section{Interrogative understanding}

Most recent inquiry into the nature of interrogative understanding focuses on examining patterns in how we ascribe this epistemic state (both to others and to ourselves). Typically, these examinations proceed by considering a set of hypothetical or real scenarios to assess whether some subject $\mathrm{S}$ in those circumstances could plausibly be said to have interrogative understanding of some fact $p .{ }^{1}$ The central assumption underlying this approach is that our evaluations of the sort ' $\mathrm{S}$ understands why $p$ ' can shed light on which conditions $S$ must satisfy in order to have interrogative understanding of $p$. Thus, it is generally assumed that we can gain explanatory purchase on what is required to understand, say, why crows fly by investigating the circumstances under which we ascribe understanding of why crows fly to others or to ourselves.

In this paper, I will take this approach for granted. That is, I assume that we can inquire into the nature of interrogative understanding by attending to how we ascribe this epistemic state. Indeed, the arguments I develop in this paper will rely on this precise methodology. However, it is noteworthy that attempts to define the nature of interrogative understanding via an analysis of our attributions (or retractions) of this epistemic state have proven notoriously divisive. Parties to these disputes diverge on whether interrogative understanding is factive, and on the relationship of this epistemic state to propositional knowledge, epistemic justification, and luck (for reviews: Brun et al. 2016; Gordon 2017; Hannon forthcoming). For current purposes, I set all such debates aside. My aim in what follows will be to make headway in inquiry about the nature of interrogative understanding without taking sides on any such controversial matters. To do so, I will focus on a pattern of interrogative under-

\footnotetext{
1 For some illustrative examples of this approach, see the bibliography in Brun et al. (2016), Gordon (2017), and Hannon (forthcoming).
} 
standing ascriptions that has been largely neglected in the recent literature: namely, their contrast-sensitivity. In the next section, I introduce an example of the contrastsensitivity of interrogative understanding that will guide discussion for the remainder of this paper. But before proceeding, it will be useful to emphasise some further points of agreement in the literature on interrogative understanding.

Understanding why $p$ is widely regarded to be a (relatively) cognitively demanding epistemic state. To establish this claim, Pritchard (2009) uses an example which seems to show that interrogative understanding is more demanding than knowledge:

Suppose that I understand why my house burned down, know why it burned down, and also know that it burned down because of faulty wiring. Imagine further that my young son asks me why his house burned down and I tell him. He has no conception of how faulty wiring might cause a fire, so we could hardly imagine that merely knowing this much suffices to afford him understanding of why his house burned down. Nevertheless, he surely does know that his house burned down because of faulty wiring, and thus also knows why his house burned down.

(Pritchard 2009, p. 38)

Building on this proposal, Pritchard argues that interrogative understanding is a kind of cognitive achievement that is creditable to the exercise of certain fairly elaborate cognitive abilities, and that this sets interrogative understanding apart from knowledge. Hills (2015) argues along similar lines, using the following example to motivate this idea:

Suppose that you know why giving money to charity is right, namely because we owe assistance to the very needy. You were told this by your parents. You understand what the statement means. You believe it and it is true. You have formed it in the right way to have knowledge (by testimony from sources you rightly believe to be reliable). But you won't necessarily yet have the abilities to make accurate judgements about other, similar cases-where you are aware that your sacrifice would be more significant, or that the needs you could meet are not pressing. And so on.

(Hills 2015, pp. 669-670)

Hills takes this example to show that interrogative understanding, unlike knowledge, involves a distinctive set of cognitive abilities. She develops this claim in terms of the popular view that interrogative understanding requires a certain 'grasp' of a body of information-which she explains as follows:

The best way of thinking of it is by an analogy with grasping a ball or cup of tea or similar. If you grasp a ball, you have it under your control. You can manipulate it, move it, turn it round, and so on [...]

(Hills 2015, p. 663)

Hills develops this analogy in terms of the claim that understanding why $p$ requires a form of cognitive control. That is, a set of abilities for manipulating the relationship between $p$ and some other proposition, $r$, which enables one to, among other things, explain $p$ in terms of $r$, and conclude $p$ from the belief that $r$. 
There has been much controversy on whether the examples offered by Pritchard and Hills succeed in driving a wedge between knowledge and interrogative understanding. ${ }^{2}$ Nevertheless, many accept that these and other similar kinds of examples suffice to demonstrate that interrogative understanding is a (relatively) cognitively demanding epistemic state. And like Hills, others have sought to flesh out this claim in terms of the idea that interrogative understanding involves a distinctive 'grasp' of a body of information (see, e.g., Kvanvig 2003; De Regt and Dieks 2005; Grimm 2014; Strevens 2016; Elgin 2017; Khalifa 2017). Now, despite there being little to no consensus on how to best characterise the notion of 'grasp', many agree that it is intricately linked to capacities for counterfactual reasoning. ${ }^{3}$ According to this view, if S understands why $p$, then $\mathrm{S}$ is sensitive not just to how things are in the world with respect to $p$, but also to how things could have been if certain factors had been different. Thus, if $\mathrm{S}$ understands why $p$, and that $r$ is why $p$, then S would not only correctly evaluate that $r$ engenders $p$, but also how changes in $r$ would affect $p$ in various possible ways.

The putative link between interrogative understanding and counterfactual reasoning will prove important for our purposes. As already mentioned, my aim is to argue that Ternary provides the best account of three important patterns of interrogative understanding ascriptions: their contrast-sensitivity, gradability, and their sensitivity to the perceived degree of epistemic demandingness of different contexts. However, as I later explain (Sect. 6), all such features may seem to be aptly accounted for in terms of the counterfactual reasoning abilities purportedly involved in interrogative understanding. This raises the question of whether Ternary does indeed afford any novel insight. I raise this issue here at the outset in order to anticipate an important objection that merits attention. But, to simplify discussion of the main arguments I develop for Ternary, I set this issue aside for now. With these considerations in mind, let us now turn to the example of contrast-sensitivity of interrogative understanding that will guide discussions in this paper.

\section{Talking about Soy}

To illustrate the contrast-sensitivity of interrogative understanding ascriptions, consider the following example.

Soy: Anna and Patrick are analysing data on the performance of the soy industry this year. Both come to understand why this sector of the economy has remained stable; however, they come to understand as much after considering distinct pieces of evidence. Patrick comes to understand why the soy industry remained stable after learning about E1 (see below), whereas Anna does so after learning about E2:

E1 Despite what was previously announced, the government decided against providing additional subsidies to soy producers this year. As a result, the soy industry did not grow.

\footnotetext{
2 See, e.g., Kelp (2015), Sliwa (2017) and Khalifa (2017, ch. 3); for a discussion: Ross (2020). I will remain neutral on this dispute here.

${ }^{3}$ For some elaborations of this view, see, e.g., Grimm (2014), Hills (2015, pp. 667-668), Strevens (2016) and Khalifa (2017, chs. $1 \&$ 2).
} 
E2 Despite the sharp decrease in China's demand for soy, South Korea has intervened to help the industry by buying up all the excess of the grain at the original price. As a result, the soy industry did not slump.

As stipulated above, both Patrick and Anna understand why the soy industry remained stable this year. Thus,

(i) Patrick understands why the soy industry remained stable; and

(ii) Anna understands why the soy industry remained stable.

Now, consider what might seem a trivial question: does Patrick understand why the soy industry remained stable? As stipulated above, after learning about E1, he comes to understand as much. However, it is patently clear that Patrick does not understand this in quite the same way as Anna (who comes to understand this after considering a distinct body of evidence E2). So, there is at least one sense in which it seems plausible to say that

(ia) Patrick does not understand why the soy industry remained stable.

And note that we can make similar considerations regarding Anna. It is clear that Anna may plausibly be said to understand why the soy industry remained stable after learning about E2; but, it is also clear that she does not understand this in quite the same way as Patrick (who understands as much after learning about E1). Thus, in at least one sense,

(iia) Anna does not understand why the soy industry remained stable.

How do we explain this shift in evaluations from (i) to (ia) and from (ii) to (iia)? One potential explanation appeals to the idea that interrogative understanding is gradable. It is in this sense that a subject $\mathrm{S}$ might sensibly be said to understand why $p$ to a greater or lesser degree than someone else. For example, consider how we might reasonably say that a 7-year-old understands why the Earth orbits the Sun to a lesser degree than an undergraduate Physics student-who presumably has more information about the astronomical facts that make it the case that the Earth orbits the Sun. Moreover, note how we can iterate this line of thinking and say that the undergraduate student will understand this astronomical fact less than a Astrophysics professor. This professor may, in turn, understand this less than an expert on the Earth's rotation-and so on and so forth.

Our recognition that interrogative understanding comes in degrees plays a central role in how we attribute this epistemic state. For instance, note how it might be sensible to claim that a 7-year-old child understands why the Earth orbits the Sun in a classroom setting, and yet deny she understands as much in the context of an undergraduate Astrophysics seminar. Now, this doesn't mean that we thereby judge that the child doesn't at all understand why the Earth orbits the Sun. Rather, this retraction is due to our recognition that one has to satisfy more demanding conditions to be attributed interrogative understanding of this fact in the latter context. Likewise, note that even if it is reasonable to claim that an undergraduate Physics student understands why the Earth revolves around the Sun in the Astrophysics seminar, it might still be appropriate to retract this attribution in the context where a panel of Astrophysics experts is discussing the rotation of the Earth. Again, this is not to say that the student completely fails 
to understand why the Earth revolves around the Sun. Rather, it is due to an implicit recognition that this latter context is more epistemically demanding and that the student does not meet the standards to be attributed interrogative understanding there.

Returning now to our protagonists Patrick and Anna. Can we explain the shift from (i) to (ia) and from (ii) to (iia) in terms of the increasing epistemic demandingness of the context? I propose that we cannot. After all, what seems to best explain our retraction of interrogative understanding attributions to Patrick and Anna is that we take there to be a fundamental difference in the kind of epistemic state we attribute to each of them (not the degree of such epistemic states). To appreciate this fact, let's stipulate that Patrick and Anna understand why the soy industry remained stable to the same degree. (Let's say they both understand this to a degree d1.) Even if we stipulate as much, I take it there is still one sense in which Patrick can be said to understand why the soy industry remained stable whereas Anna does not (namely, an understanding in terms of E1). Likewise, I hold that there is some sense in which we can say that Anna understands why this sector of the economy remained stable which Patrick fails to understand (namely, an understanding in terms of E2). Thus, appealing to the gradability of interrogative understanding and to facts about how the demandingness of different context governs our attributions of this epistemic state fails to account for the shift in evaluations we find from (i) to (ia) and from (ii) to (iia).

I propose that we can begin to gain insight into what is going on in the case of Soy when we restate our interrogative understanding attribution to Patrick as follows:

(iii) Patrick understands why the soy industry remained stable rather than grow.

Construing (i) in terms of (iii) pinpoints the specific sense in which Patrick can be said to understand why the soy industry remained stable. Moreover, this way of rephrasing Patrick's epistemic state clarifies in which sense he can be said to not understand as much:

(iv) Patrick does not understand why the soy industry remained stable rather than slump.

This seems plausible. After all, the information Patrick possesses only rules out the scenario of growth in the soy industry-not the scenario where it slumped. By restating (i) and (ia) in terms of (iii) and (iv), we can see that whether Patrick can be said to understand why the soy industry remained stable will vary depending on which alternative outcome (growing vs. slumping) is under consideration. Thus, the shift in our evaluations from (i) to (ia) is a clear case of contrast-sensitivity.

Similar considerations are apt to explain the shift in our evaluation of Anna's epistemic state. We can restate the claim that Anna understands why the soy industry remained stable as:

(v) Anna understands why the soy industry remained stable rather than slump.

Furthermore, I contend that we can aptly restate the claim that Anna does not understand why the soy industry remained stable as follows:

(vi) Anna does not understand why the soy industry remained stable rather than grow.

This suggests that our evaluation of whether Anna has interrogative understanding about the soy industry's performance is sensitive to the alternative outcome we are 
considering. Thus, our evaluation of whether Anna understands why the soy industry remained stable is also contrast-sensitive.

In sum, the considerations in this section lend support to the idea that at least some interrogative understanding ascriptions of the form ' $\mathrm{S}$ understands why $p$ ' are contrast-sensitive. ${ }^{4}$ This pattern of variation in ascriptions of interrogative understanding are puzzling. After all, we typically interpret claims of the form ' $S$ understands why p' as outright ascriptions of interrogative understanding. That is, we often take attributions of understanding-why to be invariant with respect to which alternatives to $p$ we consider. So, if I claim to understand why Pete ate an apple (because he was hungry), presumably this is unaffected by whether I understand why he ate the banana or the pizza instead. But, the example of Soy provides a clear case where attributions of interrogative understanding are indeed sensitive to such factors. In what follows, I assess the implications of this proposal for theorising about the nature of interrogative understanding.

\section{Ternary}

As already stated, Ternary is the thesis that 'understands why' expresses a threeplace epistemic relation between a subject, a fact $p$, and an alternative to $p, q$. More schematically:

Ternary 'Understands why' posits a three-place epistemic relation of understanding-why between a subject $\mathrm{S}$, a fact $p$, and an alternative to $p, q$-i.e., $\mathrm{U}-$ $\operatorname{why}_{(S, p, q)}$.

In this section, I first show that Ternary can account for the contrast-sensitivity of interrogative understanding ascriptions. I then demonstrate that Ternary offers resources with which to make sense of two other notable patterns in our attributions of this epistemic state: their gradability and their sensitivity to the epistemic demandingness of different contexts. In light of these considerations, I propose that there is at least some initial support for endorsing Ternary.

As a first step to developing the arguments in this section, let's once more return to the case of Soy. As discussed in the previous section, it seems natural to claim that Patrick can both be said to understand why the soy industry remained stable and to not so understand. To make better sense of this puzzling shift in evaluations of Patrick's epistemic states, I proposed we should restate them as follows:

(iii) Patrick understands why the soy industry remained stable rather than grow; but,

(iv) Patrick does not understand why the soy industry remained stable rather than slump.

Ternary naturally accommodates for the epistemic state ascribed in both these claims. In more detail, Ternary has us read (iii) as the claim that Patrick stands in a three-place relation of understanding-why that takes as its other two arguments the fact the soy industry remained stable and the (unrealised) alternative that this sector

\footnotetext{
${ }^{4}$ I address the question of whether all ascriptions of interrogative understanding are similarly contrastsensitive in Sect. 6.
} 
of the economy actually grew. That is, Ternary suggests that (iii) posits the epistemic relation U-why (Patrick,stable, grow). Furthermore, in line with Ternary we can read (iv) as the denial that Patrick stands in another understanding-why relation that takes as its other arguments the fact that the soy industry remained stable and the (unrealised)

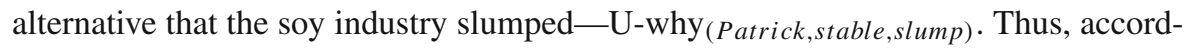
ing to Ternary, when we claim that Patrick understands why the soy industry remained stable, we judge that he satisfies the epistemic relation U-why (Patrick,stable, grow). Moreover, when we deny that Patrick understands as much, we effectively deny that he stands in the distinct epistemic relation U-why (Patrick,stable, slump) $_{\text {. }}$

Ternary also aptly accommodates for the shift in our evaluations of whether Anna understands why the soy industry remained stable. Again, recall that we can pinpoint the difference in these claims by restating them as follows:

(v) Anna understands why the soy industry remained stable rather than slump; and,

(vi) Anna does not understand why the soy industry remained stable rather than grow.

According to Ternary, we can read (v) as the claim that Anna stands in a three-place epistemic relation of understanding-why, which takes as its two other arguments the fact that the soy industry remained stable and the (unrealised) alternative that the soy industry actually slumped. That is, (v) posits the epistemic relation U-why (Anna,stable,slump). And according to Ternary, (vi) can be read as the denial that Anna stands in an understanding-why relation to the fact that the soy industry remained stable and the (unrealised) alternative that the soy industry

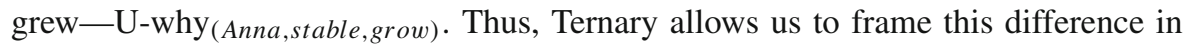
Anna's epistemic states more clearly in terms of two distinct epistemic relations: Uwhy $_{(\text {Anna,stable,slump) }}$ and U-why(Anna,stable,grow). So when we say that Anna does understand why the soy industry remained stable, we have the former epistemic relation in mind; and when we deny she understands as much, we have the latter epistemic relation instead.

In sum, Ternary aptly captures the contrast-sensitivity in our attributions of interrogative understanding to Patrick and Anna. Now, to the extent that we can regard these interrogative understanding ascriptions to be genuinely contrast-sensitive, I contend that these considerations offer a first line of support to Ternary. For the remainder of this section, I offer two further reasons to endorse this thesis.

A second welcome theoretical upshot of Ternary is that it offers a simple explanation for the gradability of interrogative understanding ascriptions. As previously noted, this feature of our attributions of interrogative understanding is made evident in how a person might be said to understand why $p$ to a greater or lesser degree than another. For example, it may be reasonable to judge that a 7-year-old child understands why the Earth orbits the Sun to a lesser degree than an undergraduate Physics student. We can imagine that this is because we recognise that even if the child may cite facts about the Sun's gravitational pull on Earth, and how this prevents the Earth from drifting off into space, they would probably fail to understand why this gravitational pull doesn't just cause the Earth to collide into the Sun. And insofar as we can expect that the Physics student will grasp that it is the orbital velocity of the Earth that prevents it from colliding into the Sun, she can then reasonably be said to have fuller understanding of why the Earth orbits the Sun. Elaborating on this example in terms of Ternary suggests 
the following description of the child's and the Physics student's respective epistemic states:

(vii) The child understands why the Earth orbits the Sun rather than drift off into space.

(viii) The Physics student understands why the Earth orbits the Sun rather than drift off into space, or collide into the Sun.

This way of fleshing out the epistemic states of the child and the Physics student provides a straightforward explanation of their differing degrees of interrogative understanding about the Earth's orbit around the Sun. The child understands this to a lesser degree because she cannot rule out the possibility that the Earth just collides into the Sun. And it is the Physics student's understanding of why this doesn't happen that explains why she can reasonably be attributed a greater degree of understanding of this fact.

More generally, the above considerations suggest that the degree to which a person can be said to understand why $p$ is a direct function of the number of alternatives to $p$ she can rule out. This account also allows us to explain why an Astrophysics professor can be said to have a much greater degree of understanding of why the Earth orbits the Sun than the undergraduate Physics student. In particular, we can expect that the Professor's expertise will enable her to eliminate a greater number of other alternatives, beyond those that the undergraduate is able to. For instance, we can plausibly say that the professor will understand why the Earth orbits the Sun rather than drift off into space, collide into the Sun, go back and forth only half-way around the Sun, etc.

A third and last welcome theoretical upshot of Ternary is that it helps to make sense of how the perception of epistemic demandingness of different contexts governs interrogative understanding ascriptions. To explain, recall once more how it can be natural to say that a 7-year-old can understand why the Earth revolves around the Sun within a classroom setting, yet to deny this attribution in a university Astrophysics seminar. A plausible explanation for this retraction appeals to the idea that we can measure degrees of interrogative understanding of $p$ as a function of the number of contrasts to $p$ one can rule out. In line with this idea, it is plausible to claim that the epistemic demandingness of a given context will be a direct function of the number of contrasts to a fact $p$ one must rule out in order to be attributed interrogative understanding of $p$. Thus, the reason a 7-year-old can be attributed understanding of why the Earth revolves around the Sun within a classroom setting is that she can rule out at least some quite simple alternatives to this fact-e.g., that the gravitational pull of the Sun does not allow the Earth to shoot off into space. However, it is sensible to expect that one must rule out a greater number of more elaborate alternatives in order to be attributed interrogative understanding of this fact in an Astrophysics seminare.g., the alternative outcome that the Earth just collides into the Sun. Likewise, to be attributed interrogative understanding of this fact in the context where a panel of experts is discussing the Earth's orbit around the Sun, one would be required to be able to rule out an even further number of alternatives, such as the fact that the Earth does not just go back and forth only half-way around the Sun-and so and so forth for more (or less) epistemically demanding contexts. 
The observations in this section thus provide some initial support for Ternary. However, they do not entail this thesis. For instance, it is an entirely open possibility that the contrast-sensitivity of interrogative understanding can be accounted for in terms of Binary-i.e., the idea that we can analyse interrogative understanding attributions in terms of a two-place relation of understanding-why between a subject and a given fact. In the following section, I assess this proposal. I first discuss the three most plausible readings Binary in which this thesis could be said to account for interrogative understanding ascriptions featuring "rather than" clauses. The readings discussed are modelled on proposals from the relevant literature that attempt to explain the contrast-sensitivity of claims that feature other philosophical concepts (e.g., 'knowledge', 'reasons', and 'explains'). After introducing these distinct readings of Binary, I then explain why there are good reasons to reject each one of them.

\section{Binary and contrast-sensitivity}

\subsection{Binary-gradable}

One seemingly promising way to account for claims of the sort ' $\mathrm{S}$ understands why $p$ rather than $q$ ' in terms of Binary is to interpret the relevant "rather than" clauses to mean "more than". On this proposal, for $\mathrm{S}$ to understand why $p$ rather than $q$, $\mathrm{S}$ must stand in the epistemic relation $\mathrm{U}-\mathrm{why}_{(S, p)}$ to a given degree $\mathrm{d} 1$, and stand in the epistemic relation $\mathrm{U}$-why $(S, q)$ to a different degree $\mathrm{d} 2$, where $\mathrm{d} 1>\mathrm{d} 2$. I denominate this view 'Binary-gradable' 5 'More schematically:

Binary-gradable $\mathrm{S}$ understands why $p$ rather than $q$ iff $\mathrm{S}$ understands why $p$ (U-why $_{(S, p)}$ ) more than she understands why $q$ (U-why $(S, q)$ ).

Binary-gradable relies on the natural idea that some people can be said to understand why $p$ to a greater or lesser degree than others. Given that interrogative understanding is indeed gradable, then Binary-gradable can be said to account for at least the following interrogative understanding ascriptions from Soy:

(iii) Patrick understands why the soy industry remained stable rather than grow this year.

(v) Anna understands why the soy industry remained stable rather than slump this year.

In line with Binary-gradable, we can read (iii) as the claim that Patrick understands why the soy industry remained stable more than he understands why it grew this year. This seems plausible. Since Patrick does understand to at least some degree why the soy industry remained stable (as stipulated in Soy), and that he simply does not understand at all why it grew (since it didn't), then he will of course understand the former to a greater extent than the latter. Thus, we can say that Patrick stands in the epistemic relation $\mathrm{U}$-why (Patrick,stable $)_{\text {to }}$ to given degree $\mathrm{d} 1$, and to the relation $\mathrm{U}$ why $_{(\text {Patrick, grow) }}$ to degree d2, where d1 > d2. Similarly, Binary-gradable suggests

\footnotetext{
5 Binary-gradable models similar attempts to account for the contrast-sensitivity of reasons claims (Snedegar 2017, p. 26) 
that (v) can be read as the claim that Anna understands why the soy industry remained stable more than she understands why it slumped. Again, this seems right: since Anna has some degree of understanding of why it remained stable (as stipulated), yet no understanding of why the soy industry slumped, then it would seem right to say that she understands the former more than the latter. In other words, i.e., U-why (Anna,stable) to degree $\mathrm{d} 3$, and U-why (Anna,slump) to degree $\mathrm{d} 4$, where $\mathrm{d} 3>\mathrm{d} 4$.

So far so good. But, even if Binary-gradable might seem promising at first, I will now argue that this view runs into trouble. This problem arises because this view posits a strict relation between the contrast-sensitivity of interrogative understanding ascriptions and differences in degrees of understanding. Thus, a natural corollary of Binary-gradable is that the negation of ' $\mathrm{S}$ understands why $p$ rather than $q$ ' either implies that $\mathrm{S}$ has a same degree of understanding-why of both $p$ and $q$, or that $\mathrm{S}$ actually understands why $q$ more than she understands why $p$. To see why positing this strict relation between contrast-sensitivity and degrees of understanding causes trouble for Binary-gradable, consider again the following evaluation of Patrick and Anna's epistemic states from Soy:

(iv) Patrick does not understand why the soy industry remained stable rather than slump.

(vi) Anna does not understand why the soy industry remained stable rather than grow.

The challenge for Binary-gradable is that on this view, (iv) is to be read either as:

(iva) Patrick understands (to degree d1) why the soy industry remained stable to a same degree (to degree $\mathrm{d} 1$ ) as to why it slumped; or

(ivb) Patrick understands (to degree d2) why the soy industry slumped more than he understands (to degree $\mathrm{d} 1$ ) why it remained stable $(\mathrm{d} 2>\mathrm{d} 1)$.

Similarly, Binary-gradable has us read (vi) as either:

(via) Anna understands (to degree $\mathrm{d} 3$ ) why the soy industry remained stable to a same degree (to degree $\mathrm{d} 3$ ) as to why it grew; or

(vib) Anna understands why the soy industry grew (to degree d4) more than she understands (to degree $\mathrm{d} 3$ ) why it remained stable $(\mathrm{d} 4>\mathrm{d} 3)$.

Note that all of (iva), (ivb), (via), and (vib) are ultimately implausible. The correct explanation for (iv) and (vi) is that Patrick and Anna have a restricted amount of information about the soy industry's performance, and as such they do not understand the stability of the soy industry with respect to every potential scenario (growth/slump). This underscores the fact that there are distinct ways of understanding the performance of a given sector of the economy, and that these make salient incompatible sets of contrasts (stable rather than grow, or stable rather than slump). By forcing a reading of "rather than" clauses purely in terms of degrees of understanding, Binary-gradable fails to capture this subtlety in our ascriptions of understanding-why. 


\subsection{Binary-conjunction}

A second natural way to try to account for occurrences of "rather than" clauses in terms of Binary is to interpret these clauses to mean "and not". 6 This amounts to the proposal that for a subject $\mathrm{S}$ to understand why $p$ rather than $q$, she must stand in two distinct epistemic relations: U-why $(S, p)$ and U-why ${ }_{(S, n o t-q)}$. I denominate this view 'Binary-conjunction'? More schematically:

Binary-conjunction $\mathrm{S}$ understands why $p$ rather than $q$ iff $\mathrm{S}$ understands why $p$ $\left.\operatorname{(U-why}_{(S, p)}\right)$ and why not- $q\left(\mathrm{U}-\mathrm{why}_{(S, n o t-q)}\right)$.

To a first approximation, Binary-conjunction seems to neatly capture the contrastsensitivity of interrogative understanding ascriptions. To see how, consider once more our evaluation of Patrick's epistemic states from Soy:

(iii) Patrick understands why the soy industry remained stable rather than grow.

(iv) Patrick does not understand why the soy industry remained stable rather than slump.

According to Binary-conjunction, (iii) can be read as the claim that Patrick understands why the soy industry remained stable and why it did not grow. This reading seems plausible. After all, Patrick knows that despite what was previously announced, the government did not provide additional subsidies to soy producers this year, making it so that the soy industry did not grow. In this sense, it is easy to see that he both understands why the soy industry remained stable (U-why (Patrick,stable) $)$, and why it did not grow (U-why (Patrick,not-grow) $)$. Furthermore, in line with Binary-conjunction, we can read (iv) as the claim that Patrick does not understand why the soy industry remained stable and why it did not slump. This also sounds plausible. After all, it is reasonable to deny that Patrick has understanding of why this sector of the economy did not slump.

Similar considerations appear apt to account for our evaluation of Anna's epistemic states:

(v) Anna understands why the soy industry remained stable rather than slump this year.

(vi) Anna does not understand why the soy industry remained stable rather than grow this year.

Binary conjunction suggests that we can read (v) as the claim that Anna understands why the soy industry remained stable and why it did not slump. Again, this is plausible. For, Anna has been informed that despite the sharp decrease in China's demand for soy, South Korea has intervened to help the soy industry by buying up all the excess of the grain at the original price-which prevented a slump in the sector. In this sense, Anna can be said to both understand why the soy industry remained stable

\footnotetext{
6 Binary-conjunction models similar proposals that purport to explain the contrast sensitivity of claims featuring 'explains' (Ruben 1987), 'knows' (Kallestrup 2009) and 'reason' (Snedegar 2017, p. 26).

7 Another possible formulation of this proposal is that ' $S$ understands why $p$ rather than q' means that $S$ has interrogative understanding of the complex proposition $p$-and-not- $q$. The arguments I develop in this section apply, mutatis mutandis, to this alternative reading.
} 


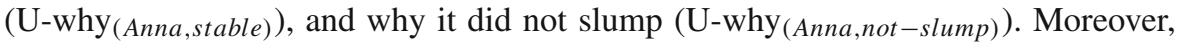
we can similarly reinterpret (vi) as the claim that Anna does not understand why the soy industry remained stable and why it did not grow. Given that Anna has insufficient information to be attributed this second epistemic state, it then makes sense to deny this conjunction of interrogative understanding ascriptions.

So, there are at least some reasons to think Binary-conjunction offers a plausible reading of interrogative understanding ascriptions featuring "rather than" clauses. However, I contend that we should reject Binary-conjunction. The problem is that on this view, understanding why $p$ and why not- $q$ is both a necessary and sufficient to understand why $p$ rather than $q$. As we will see below, there is good reason to reject this claim.

Let's begin by discussing a case where the sufficiency direction of Binaryconjunction fails. Consider the case of a skilled mathematician Mary who understands why there are an infinite number of primes. Thus, Mary satisfies the epistemic relation of U-why (Mary, infinite primes) . Now, suppose Mary also understands why it is not the case that 4 is a prime number. In this sense, we can say that Mary satisfies the epistemic relation of U-why (Mary, not-4 prime) . So, Mary both understands why there is an infinity of prime numbers, and why it is not the case that 4 is a prime number. According to Binary-conjunction, Mary would thereby understand why there is an infinite number of primes rather than 4 being a prime number. However, it is implausible to attribute this epistemic state to Mary. Thus, I contend we should reject the sufficiency direction of Binary-conjunction.

Let's now turn to the claim that understanding why $p$ and why not- $q$ is necessary for understanding why $p$ rather than $q$. Focusing on examples again helps to show why this fails. Consider the case in which Mary understands why being subjected to high levels of radioactivity would cause her harm rather than transform her into a superhero. Suppose this is because she's well aware of the dangers of radioactivity, and of the fact that there is no verified case in which radioactivity transformed people into superheroes. According to Binary-conjunction, we should thereby say that Mary both understands why being subjected to high levels of radioactivity would cause her harm (U-why (Mary,harm) ), and why it would not transform her into a superhero (U-why (Mary,not-superhero) $)$. But, suppose Mary has absolutely no idea of why radioactivity is harmful to people. It is simply not clear to her what about radioactive particles cause the terrible ailments they do-e.g., why they damage internal organs, why they cause a slow death, and what about them is so threatening. Nevertheless, note that this is entirely compatible with the possibility that Mary does indeed understand why radioactivity would cause her harm rather than transform her into a superhero. After all, she does have sufficient information showing her that there is no evidence to think otherwise. In this sense, understanding why $p$ and why not- $q$ is not necessary for understanding why $p$ rather than $q$.

These considerations show that there are good reasons to reject Binary-conjunction. As the examples above illustrate, understanding why $p$ and why not- $q$ is neither necessary nor sufficient to understand why $p$ rather than $q$. As such, Binary-conjunction cannot account for interrogative understanding ascriptions featuring a "rather than" clause - and so it fails to capture the contrast-sensitivity of interrogative understanding. 


\subsection{Binary-question}

A third way one might try to account for the contrast-sensitivity of interrogative understanding is to take claims of the sort ' $\mathrm{S}$ understands why $p$ rather than $q$ ' to posit a link between a subject and a question. ${ }^{8}$ Thus, the evaluation that $S$ understands why $p$ rather than $q$ would effectively amount to the claim that $\mathrm{S}$ satisfies the epistemic relation $\mathrm{U}$-why $(S, Q)$, where $\mathrm{Q}$ is the relevant question 'why $p$ rather than $q$ ?'. I dub this proposal 'Binary-question'. More schematically:

Binary-question $\mathrm{S}$ understands why $p$ rather than $q$ iff $\mathrm{S}$ understands 'why $p$ rather than $q$ ?' (U-why $(S, Q))$.

This proposal needs to be clarified. Binary-question is not the claim that if $\mathrm{S}$ understands why $p$ rather than $q$, then S linguistically understands this question. Instead, it is the claim that if $\mathrm{S}$ stands in an understanding-why relation to "why $p$ rather than $q$ ?', then $\mathrm{S}$ understands that $\mathrm{A}$ is an answer to this question.

To a first approximation, Binary-question seems apt to explain the contrastsensitivity of interrogative understanding. To see how, recall Patrick's epistemic states from Soy:

(iii) Patrick understands why the soy industry remained stable rather than grow.

(iv) Patrick does not understand why the soy industry remained stable rather than slump.

In line with Binary-question, (iii) can be read as the claim that Patrick satisfies the epistemic relation of understanding-why with respect to the question 'why did the soy industry remain stable rather than grow?'-i.e., Uwhy (Patrick, 'why stable rather than grow?'). This amounts to the claim that Patrick understands that $\mathrm{A}$, where $\mathrm{A}$ is an answer to this particular question. This seems plausible. After all, we can imagine that Patrick does understand that the fact the government failed to give additional subsidies to the soy industry is why this sector of the economy remained stable rather than grow. Moreover, it is reasonable to expect that Patrick would fail to understand an answer to the question 'why did the soy industry remain stable rather than slump?'. After all, the only information he has concerns the question of why the soy industry did not grow. Thus, it seems plausible to read (iv) as the claim that Patrick fails to satisfy the epistemic relation of understanding-why to the question 'why did the soy industry remain stable rather than slump?'-U-why ${ }_{(\text {Patrick, 'why stable rather than slump?') }}$. (I trust the reader will appreciate that similar considerations appear apt to account for the contrast-sensitivity of Anna's epistemic states in Soy).

These brief considerations give us at least some reason to think that Binary-question can indeed account for the contrast-sensitivity of interrogative understanding. However, I contend that this is a mistake. The main problem with Binary-question is that it seeks to reduce interrogative understanding to another kind of epistemic state: namely, propositional understanding. The latter is the epistemic state ascribed by claims of the form 'S understands that $p$ ', such as 'Patrick understands that the government did

\footnotetext{
${ }_{8}$ Binary-conjunction is modelled on similar proposals attempting to account for the contrast-sensitivity of claims featuring 'knows' (Masto 2010).
} 
not provide additional subsidies to the soy industry' or 'Anna understands that South Korea intervened and bought up all the excess soy'. To see why this is problematic, recall once more our protagonist Anna from Soy. Suppose Anna has a 10-year-old daughter, Mary, who is very curious. One day, Anna tells Mary that although China stopped importing soy, South Korea bought up all the grains which China did not buy at the same price. Imagine that Anna then adds that this answers the question of why the soy industry remained stable rather than slump. Now, note how it is plausible to say that Mary may indeed understand that the fact South Korea bought up all the excess grain is an answer to the question "why did the soy industry remain stable rather than slump?'. Nevertheless, it is entirely possible that Mary may still fail to understand why the soy industry remained stable rather than slump. After all, Mary may very well fail to fully understand the economic facts that make it the case that South Korea's intervention prevents the soy industry from collapsing. In this sense, we have a case in which Mary understands that some fact A is the answer to 'why did the soy industry remain stable rather than slump?', yet where she fails to understand why the soy industry remained stable rather than slump.

This example effectively demonstrates that attempts to reduce interrogative understanding of $p$ to propositional understanding of an answer to 'why p?' fail. As such, I contend that Binary-question cannot adequately account for the contrast-sensitivity of interrogative understanding ascriptions.

\subsection{Taking stock}

I have discussed three readings of Binary under which this thesis may appear to account for the contrast-sensitivity of interrogative understanding ascriptions. According to these readings, we can analyse interrogative understanding ascriptions featuring "rather than" clauses in terms of two-place epistemic relations of understanding-why holding between a subject and some fact. I then showed why each of these readings of Binary fails to do so. Given these shortcomings, I contend there are good reasons to think Binary cannot account for the contrast-sensitivity of interrogative understanding.

Now, it is clear that the arguments in this section are not decisive. I am not claiming that there is no way of fleshing out Binary such that it can account for the contrastsensitivity of interrogative understanding ascriptions. However, I contend that no such reading has been offered in the literature, nor have there been any analogous views proposed to account for the contrast-sensitivity of other philosophically relevant concepts. Moreover, since Ternary naturally accommodates for the contrast-sensitivity in interrogative understanding attributions, I contend that we should prefer Ternary to Binary. 


\section{Objections}

\section{On counterfactual reasoning}

As discussed at the outset (Sect. 2), many take interrogative understanding to involve counterfactual reasoning abilities. According to this view, if $\mathrm{S}$ understands why $p$, and that $r$ is why $p$, then $\mathrm{S}$ would not only correctly evaluate that $r$ engenders $p$, but also how changes in $r$ would affect $p$ in various possible ways. It is in this sense that Grimm (2014) claims that understanding why $p$ requires 'seeing' or 'grasping' both the causal dependencies of $p$ and the modal relatedness of such causal factors to $p$. Thus, on Grimm's view, to understand why crows fly is to register not only the causal dependencies that make it the case that crows fly, but also to have an elaborate modal sense of how changes in those causal factors would affect whether or not crows fly. Khalifa (2017) develops an alternative (more minimalist) view of the link between interrogative understanding and counterfactual reasoning. His proposal is that understanding of why $p$ is intricately linked to a capacity to correctly evaluate explanations for why $p$, which, in turn, requires the ability to evaluate some informative counterfactuals. And on a similar note, Hills (2015, pp. 667-668) suggests that understanding why $p$ requires a form of 'cognitive control' of an explanation for $p$, which, when well-developed, involves abilities to answer questions about what would happen to $p$ if certain relevant factors had been different.

This well-established connection between interrogative understanding and counterfactual reasoning proves important for current purposes. On a plausible reading of this view, if $\mathrm{S}$ understands why $p$, then $\mathrm{S}$ would be poised to correctly evaluate what would have to happen for an unrealised alternative to $p, q$, to obtain instead, and why $q$ did not obtain after all. In effect, this means that $S$ would thus understand why $p$ rather than $q$. In this light, it is worth asking: could we not capture the contrast-sensitivity of interrogative understanding entirely in terms of this link to counterfactual reasoning? To substantiate this idea, recall the example of Patrick in Soy once more. As we saw, this case stipulates that

(i) Patrick understands why the soy industry remained stable.

because he learned that the government did not provide additional subsidies to soy producers this year, and so this sector of the economy did not grow as expected. Yet, it is also reasonable to say that

(ia) Patrick does not understand why the soy industry remained stable.

insofar as he does not understand this in the same way that Anna does. Now, note that we can aptly characterise this shift in our evaluations of Patrick's epistemic states as a function of the counterfactual claims we can reasonably expect him to correctly evaluate. For example, Patrick is presumably in a position to correctly evaluate which conditions would have to have been in place for the soy industry to have grown as expected. But, it is implausible to say that Patrick's epistemic state would allow him to engage in counterfactual reasoning about how the soy industry might easily have slumped instead of remaining stable (in the way that Anna could). Thus, the evaluation that 
(iii) Patrick understands why the soy industry remained stable rather than grow

can be aptly explained in terms of our expectation that Patrick would be able to engage in counterfactual reasoning about the possibility that the soy industry could have grown if additional subsidies had been given; and the evaluation that

(iv) Patrick does not understand why the soy industry remained stable rather than slump

is presumably explained by our expectation that he would not be in a position to evaluate relevant counterfactuals on what could have led the soy industry to slump.

These considerations raise the question of whether Ternary is indeed an informative thesis. After all, one of the main motivations for Ternary is that this thesis seems apt to capture the contrast-sensitivity of interrogative understanding ascriptions (Sect. 4). But, if we can explain this feature in terms of abilities for counterfactual reasoning, then would Ternary not just be a restatement of the idea that interrogative understanding enables one to evaluate relevant counterfactuals? ${ }^{9}$ To press this concern some more, note that we can also recast the two other arguments given for Ternary in terms of this link to counterfactual reasoning. For example, the gradability of interrogative understanding could presumably also be explained as a function of the kinds and number of counterfactual claims one could evaluate. So, the 7-year-old child understands why the Earth orbits the Sun to a lesser degree than an undergraduate Physics student because the former can evaluate fewer and less complex counterfactuals than the student. Furthermore, this way of characterising the gradability of interrogative understanding can help explain what guides our evaluation of the epistemic demandingness of different contexts. For, we would presumably expect that attributions of interrogative understanding of the Earth's orbit in, say, a classroom setting would depend on which kinds of counterfactual claims about orbital rotation one would be expected to correctly evaluate in that context.

Reply: I contend that upon closer scrutiny, Ternary proves more adequate to explain features of interrogative understanding than explanations in terms of abilities for counterfactual reasoning. To develop this claim, it is first useful to show how the abilities for counterfactual reasoning involved in interrogative understanding are the same, regardless of whether we express this epistemic state in terms of Ternary or Binary. To explain, let's focus on Binary-conjunction (see Sect. 5.2). ${ }^{10}$ As previously discussed, Binary-conjunction has us read the claims

(iii) Patrick understands why the soy industry remained stable rather than grow and

(iv) Patrick does not understand why the soy industry remained stable rather than slump.

as the claims that Patrick understands why the soy industry remained stable and why it did not grow, but that he does not understand why the soy industry did not

\footnotetext{
9 I would like to thank an anonymous reviewer for suggesting I discuss this objection.

10 The arguments I offer here can be developed, mutatis mutandis, for Binary-gradable and Binary-question. But for current purposes, all we need is to show that these arguments work for Binary-conjunction.
} 
slump. Now, given that interrogative understanding involves abilities for counterfactual reasoning, it is instructive to ask: what kinds of counterfactual claims is Patrick poised to correctly evaluate? To answer this question, let's focus on some of the accounts detailed in the beginning of this section.

On Grimm's account, Patrick would presumably 'see' or 'grasp' that the failure to provide additional subsidies is the main factor that led the soy industry to remain stable, and that giving such subsidies would have led the soy industry to have grown instead. However, Patrick is presumably not in a position to 'see' or 'grasp' what causal factors were likely to have led the soy industry to have slumped (in the way Anna is). Likewise, on Khalifa's proposal Patrick would be in a position to run some informative counterfactuals about what would have to have happened for the soy industry to have grown, but not on what conditions were likely to have led this sector of the economy to have slumped. And in line with Hill's proposal, Patrick would have cognitive control over the explanation for why the soy industry remained stable, which presumably allows him to draw the conclusion about what would have to have been in place for it to have grown - but not about what would have to have been in place for it to have slumped.

Thus, on all these accounts, Patrick can reasonably be expected to correctly evaluate counterfactuals about the possibility that the soy industry could have grown if additional subsidies had been given; however, he is plausibly not in a position to evaluate relevant counterfactuals on what could have led the soy industry to slump. Now, note that these are presumably the very same kinds of counterfactual claims involved in Patrick's epistemic state when this is framed in terms of Ternary. After all, the fact that Patrick satisfies the relation U-why (Patrick,stable, grow) but not U-why (Patrick,stable, slump) effectively means that he can carry out counterfactual reasoning about how the soy industry could have grown, but not about the alternative scenario about how this sector could have slumped. However, if the abilities for counterfactual reasoning are the same, regardless of whether we express Patrick's epistemic state in terms of Binary-conjunction or Ternary, then Ternary cannot be a mere restatement of the link between interrogative understanding and abilities for counterfactual reasoning. After all, as argued for in detail in previous sections, Binary-conjunction and Ternary are incompatible. Thus, even if satisfying epistemic relations of the form $\mathrm{U}$-why $(S, p, q)$ enable one to evaluate relevant counterfactuals about $p$ and $q$, this does not mean that this epistemic relation is reducible to such abilities for counterfactual reasoning.

Furthermore, I contend that since the link to counterfactual reasoning is invariant under Ternary and Binary-conjunction, and given there are good reasons to reject Binary-conjunction, then Ternary affords more insight into the nature of interrogative understanding than explanations in terms of abilities for counterfactual reasoning. To explain, note that as illustrated above with the case of Patrick, explanation of the contrast-sensitivity of interrogative understanding in terms of abilities for counterfactual reasoning is compatible with Binary-conjunction. But, given my arguments for thinking Binary-conjunction is false (Sect. 5.2), this explanation in terms of abilities for counterfactual reasoning is inadequate. After all, such an explanation will ultimately be compatible with a thesis which we have good reason to reject. Thus, an account of the contrast-sensitivity in terms of Ternary is more accurate. After all, 
it not only captures the link between interrogative understanding and counterfactual reasoning, but is also strictly incompatible with (all versions of) Binary.

Similar considerations show why there are good reasons to prefer explanations of the gradability of interrogative understanding in terms of Ternary to explanations in terms of abilities for counterfactual reasoning. Once more, the problem with the latter kind of explanation is that it is compatible with Binary-conjunction. To illustrate, consider once more the case of gradability previously discussed in which a 7-year-old-child is plausibly said to understand why the Earth orbits the Sun to a lesser degree than an undergraduate Physics student. As previously discussed (Sect. 4), we can rephrase the epistemic states of this child and the Physics student as follows:

(vii) The child understands why the Earth orbits the Sun rather than drift off into space.

(viii) The Physics student understands why the Earth orbits the Sun rather than drift off into space, or collide into the Sun.

According to the explanation in terms of abilities for counterfactual reasoning, the child understands why the Earth orbits the Sun to a lesser degree because she can correctly evaluate fewer and less complex counterfactuals than the Physics student. For example, we can expect her to correctly evaluate what would happen if the gravitational pull of the Sun faded, but not what would need to happen for the Earth to indeed collide into the Sun (as the Physics student would). Now, note that this explanation is entirely compatible with a Binary-conjunction reading of the relevant epistemic states. On this view, (vii) expresses the claim that the child understands both why the Earth orbits the Sun and why the Earth does not drift off into space. And presumably, the latter epistemic state would involve precisely the same abilities for counterfactual reasoning already identified above: namely of correctly evaluating what would happen if the gravitational pull of the Sun faded, but not what would need to happen for the Earth to collide into the Sun. These considerations effectively demonstrate that an explanation of the gradability of interrogative understanding in terms of counterfactual reasoning is compatible with Binary-conjunction. Moreover, given there is good reason to reject this thesis, then this explanation in terms of counterfactuals is thereby inadequate. Thus, I contend we should prefer an account of the gradability of interrogative understanding in terms of Ternary - as, again, this account not only aptly captures the link between interrogative understanding and counterfactual reasoning, but also is incompatible with (all versions of) Binary. (I trust the reader will see how these considerations show that Ternary is better suited to explain the sensitivity of interrogative understanding attributions to the demandingness of different contexts.)

In sum, far from being a mere restatement of the link between interrogative understanding and counterfactual reasoning, I contend that Ternary proves to be an informative thesis. For, as suggested in this section, there are good reasons to prefer explanations of the contrast-sensitivity, gradability, and context-sensitivity of interrogative understanding ascriptions in terms of Ternary over explanations in terms of abilities for counterfactual reasoning — as the latter is compatible with (at least one version of) Binary. Thus, I maintain that Ternary offers valuable resources that can help guide inquiry into the nature of interrogative understanding. 
Lastly, there is yet an additional consideration in favour of explanations in terms of Ternary. Notably, there is an ongoing and heated dispute about how to best characterise the abilities for counterfactual reasoning involved in interrogative understanding. Indeed, as pointed out earlier in this section, the approaches developed by Grimm, Khalifa and Hills offer quite different perspectives on this issue (see also Strevens 2016). Accounts in terms of Ternary are independent of such disputes, thus allowing us to provide a unified explanation of features of interrogative understanding without having to take sides on such controversial matters. This indicates another way in which Ternary proves to be more theoretically fruitful than explanations in terms of counterfactual reasoning abilities.

\section{Is interrogative understanding always contrastive?}

According to Ternary, a subject $\mathrm{S}$ never understands why $p$ simpliciter. Instead, understanding of why $p$ is always relative to some particular contrast to $p, q$. This means that whenever we say that some subject $\mathrm{S}$ understands why $p$, we actually claim that $\mathrm{S}$ understands why $p$ rather than $q\left(\mathrm{U}-\mathrm{why}_{(S, p, q)}\right)$. Now, as the examples discussed in this paper demonstrate, there are some cases in which this proposal seems apt. For instance, the contrast-sensitivity we find in the case of Soy seems to be best accounted for in terms of the idea that interrogative understanding is contrastive. But, once we move past these examples, it might seem less clear that contrastivism is always appropriate. For instance, consider a case in which a subject $\mathrm{S}$ is said to understand why 2 is a prime number. Is it appropriate to say that what we actually express in this case is that $S$ understands why 2 is a prime number rather than not? This might seem somewhat odd. After all, we don't typically make it explicit that we are attributing this latter epistemic state. So, why should we accept this outcome of Ternary?

Reply: Although it is true that attributions of interrogative understanding do not typically make it explicit that they are relative to some contrast or other, note that we also often do not make explicit the degree of interrogative understanding that we ascribe to others or ourselves. That is, we do not typically claim that ' $\mathrm{S}$ understands why $p$ to degree $\mathrm{d} 1$ '. Nevertheless, it is clear that we do evaluate the degree to which $\mathrm{S}$ will understand why $p$ when ascribing to her this epistemic state. For example, it is clear that when I say that a 7-year-old understands why the Earth revolves around the Sun, I implicitly recognise that she will understand this to a lesser degree than an undergraduate Physics student. Furthermore, note that attributions of interrogative understanding typically do not make it explicit that they are relative to a particular context. For example, when I claim that a 7-year-old understands why the Earth revolves around the Sun, I will typically attribute this epistemic state with some particular set of contexts in mind (e.g., those of a classroom setting, rather that of an Astrophysics Seminar). Yet, when I ascribe interrogative understanding to this child, I will typically not make it explicit which context I have in mind. Now, to the extent that it seems natural to consider the degree of interrogative understanding and the epistemic demandingness of different contexts as governing our attributions of interrogative understanding, even when these are not explicitly noted, it is unclear why we should not accept that 
contrasts can also govern our attributions of interrogative understanding (even if they are not explicitly noted).

\section{Concerns about triviality}

Perhaps one of the most well-accepted formulations of contrastivism in the recent philosophical literature is the idea that explanation is contrastive (see, e.g., Van Fraassen 1980). According to this view, whether some fact can plausibly be said to explain why $p$ will depend on which alternatives to $p$ we focus on. For instance, suppose I cite the fact that Peter was hungry (E) as an explanation for why Peter ate the apple. Now, it is quite reasonable to think that it is true that E explains why Peter ate the apple rather than just stay seated on the sofa; yet, it is intuitively false to say that E explains why Peter ate the apple rather than the pear, or the pizza since these different options of food could just as well have satiated Peter's hunger. In effect, this suggests that whether the fact E succeeds in explaining why Peter ate the apple will hinge on which particular class of contrasts we focus on-e.g., whether we focus on Peter's actions or on his choices of food.

The idea that explanation is contrastive in this way has important consequences for an evaluation of the arguments developed in this paper. That is because many contend that interrogative understanding is intrinsically linked to explanation. According to this view, a minimal condition for understanding why $p$ is that one have a certain set of abilities for explaining why $p$. Now, if interrogative understanding of $p$ is indeed underwritten by an ability to explain why $p$, then one might think that contrastivism about interrogative understanding is simply parasitic on the fact that explanation itself is contrastive. As such, the proposal that interrogative understanding is contrastive would not be any different from the already influential view that explanations are contrastive.

Reply: To begin, it is important to note that there is significant controversy over the idea that interrogative understanding of $p$ is indeed linked to an ability to explain why p. For instance, Lipton (2009) has famously argued that after considering Galileo's thought-experiments, one can reasonably be said to understand why the acceleration of bodies does not depend on their masses. However, as Lipton points out, it is entirely possible that one may still fail to provide an explanation for why this is the case. And Newman (2012) contends that one can have interrogative understanding of $p$ even when one has only an ability to make inferences about why some fact might explain a given explanandum - that is, even if one lacks a full-fledged explanation.

The controversy surrounding the role of explanation in interrogative understanding underscores how the arguments in this paper prove informative for future research on the nature of this epistemic state. For, on the one hand, if interrogative understanding is not intrinsically linked to an ability to explain, then the idea that interrogative understanding is contrastive offers novel insight into the nature of this epistemic state. On the other hand, if one defends interrogative understanding is indeed underwritten by an ability to explain, then the arguments in this paper can be taken as offering support for this thesis. In this way, the proposal that interrogative understanding is contrastive is far from trivial. 


\section{Conclusion}

I have been arguing for Ternary-i.e., the view that 'understands why' posits a threeplace epistemic relation of understanding-why between a subject, a fact $p$, and a contrast to $p, q$. In support of this thesis, I first demonstrated how some statements of the form ' $\mathrm{S}$ understands why $p$ ' are contrast-sensitive and that these attributions actually express claims of the form ' $\mathrm{S}$ understands why $p$ rather than $\mathrm{q}$ '. I then showed that Ternary naturally accounts for such interrogative understanding attributions, as well as two other notable patterns in how we ascribe this epistemic state. I then considered and rejected non-contrastive accounts of interrogative understanding attributions featuring "rather than" clauses. Lastly, I discussed and refuted three objections to the arguments in this paper. In light of these considerations, I contend that there are indeed good reasons to think that interrogative understanding is contrastive.

Acknowledgements Many thanks to Matteo Colombo, Insa Lawler, Lewis Ross, Justin Snedegar, and to audiences at GAP10 and the 11th Principia International Symposium for valuable discussions on this material. I am also indebted to the two anonymous reviewers for this journal for their excellent comments which have greatly improved this paper.

Open Access This article is licensed under a Creative Commons Attribution 4.0 International License, which permits use, sharing, adaptation, distribution and reproduction in any medium or format, as long as you give appropriate credit to the original author(s) and the source, provide a link to the Creative Commons licence, and indicate if changes were made. The images or other third party material in this article are included in the article's Creative Commons licence, unless indicated otherwise in a credit line to the material. If material is not included in the article's Creative Commons licence and your intended use is not permitted by statutory regulation or exceeds the permitted use, you will need to obtain permission directly from the copyright holder. To view a copy of this licence, visit http://creativecommons.org/licenses/by/4.0/.

\section{References}

Brun, G., Beisbart, C., \& Baumberger, C. (2016). What is understanding? An overview of recent debates in epistemology and philosophy of science. In S. R. Grimm, C. Baumberger, \& S. Ammon (Eds.), Explaining understanding (pp. 17-50). New York: Taylor \& Francis.

De Regt, H. W., \& Dieks, D. (2005). A contextual approach to scientific understanding. Synthese, 144(1), $137-170$.

Elgin, C. Z. (2017). True enough. Cambridge: MIT Press.

Gordon, E. C. (2017). Understanding in epistemology. In Internet Encyclopedia of Philosophy.

Grimm, S. R. (2014). Understanding as knowledge of causes. In Virtue epistemology naturalized (pp. 329-345). New York: Springer.

Hannon, M. (forthcoming). Recent work in the epistemology of understanding. American Philosophical Quarterly.

Hills, A. (2015). Understanding why. Nô̂s, 49(2), 661-688.

Kallestrup, J. (2009). Knowledge-wh and the problem of convergent knowledge. Philosophy and Phenomenological Research, 78(2), 468-476.

Kelp, C. (2015). Understanding phenomena. Synthese, 192(12), 3799-3816.

Khalifa, K. (2017). Understanding, explanation, and scientific knowledge. Cambridge: Cambridge University Press.

Kvanvig, J. L. (2003). The value of knowledge and the pursuit of understanding. Cambridge: Cambridge University Press.

Lipton, P. (2009). Understanding without explanation. In H. W. De Regt, S. Leonelli, \& K. Eigner (Eds.), Scientific understanding: Philosophical perspectives (pp. 43-63). Pittsburgh: University of Pittsburgh Press. 
Masto, M. (2010). Questions, answers, and knowledge-wh. Philosophical Studies, 147(3), 395-413.

Newman, M. (2012). An inferential model of scientific understanding. International Studies in the Philosophy of Science, 26(1), 1-26.

Pritchard, D. (2009). Knowledge, understanding and epistemic value. Royal Institute of Philosophy Supplement, 64, 19.

Ross, L. D. (2020). Is understanding reducible? Inquiry: An Interdisciplinary Journal of Philosophy, 63(2), 117-135. https://doi.org/10.1080/0020174x.2018.1562379.

Ruben, D. H. (1987). Explaining contrastive facts. Analysis, 47(1), 35-37.

Sliwa, P. (2017). Moral understanding as knowing right from wrong. Ethics, 127(3), 521-552.

Snedegar, J. (2017). Contrastive Reasons. Oxford: Oxford University Press.

Strevens, M. (2016). How idealizations provide understanding. In S. R. Grimm, C. Baumberger, \& S. Ammon (Eds.), Explaining understanding (pp. 53-65). New York: Taylor \& Francis.

Van Fraassen, B. C. (1980). The scientific image. Oxford: Oxford University Press.

Publisher's Note Springer Nature remains neutral with regard to jurisdictional claims in published maps and institutional affiliations. 